(C) 1999 The American Institute of Physics

\title{
PROGRESS IN ESTABLISHING A CONNECTION BETWEEN THE ELECTROMAGNETIC ZERO-POINT FIELD AND INERTIA
}

\author{
Bernhard Haisch ${ }^{1}$ and Alfonso Rueda ${ }^{2}$ \\ ${ }^{1}$ Solar \& Astrophysics Laboratory, Lockheed Martin, H1-12, B252, 3251 Hanover St., Palo Alto, CA 94304 \\ haisch@starspot.com \\ ${ }^{2}$ Dept. of Electrical Eng. and Dept. of Physics \& Astronomy, California State Univ., Long Beach, CA 90840 \\ arueda@csulb.edu \\ Presented at Space Technology and Applications International Forum (STAIF-99) \\ January 31-February 4, 1999, Albuquerque, NM
}

\begin{abstract}
We report on the progress of a NASA-funded study being carried out at the Lockheed Martin Advanced Technology Center in Palo Alto and the California State University in Long Beach to investigate the proposed link between the zero-point field of the quantum vacuum and inertia. It is well known that an accelerating observer will experience a bath of radiation resulting from the quantum vacuum which mimics that of a heat bath, the so-called DaviesUnruh effect. We have further analyzed this problem of an accelerated object moving through the vacuum and have shown that the zero-point field will yield a non-zero Poynting vector to an accelerating observer. Scattering of this radiation by the quarks and electrons constituting matter would result in an acceleration-dependent reaction force that would appear to be the origin of inertia of matter (Rueda and Haisch 1998a, 1998b). In the subrelativistic case this inertia reaction force is exactly newtonian and in the relativistic case it exactly reproduces the well known relativistic extension of Newton's Law. This analysis demonstrates then that both the ordinary, $\vec{F}=m \vec{a}$, and the relativistic forms of Newton's equation of motion may be derived from Maxwell's equations as applied to the electromagnetic zero-point field. We expect to be able to extend this analysis in the future to more general versions of the quantum vacuum than just the electromagnetic one discussed herein.
\end{abstract}

\section{BACKGROUND}

In July 1998 the Advanced Concepts Office at JPL and the NASA Office of Space Science sponsored a four-day workshop at Caltech on "Robotic Interstellar Exploration in the Next Century." The objective was to bring together scientists and engineers to survey the landscape of possible ideas that could lead to unmanned missions beyond the Solar System beginning within a timeframe of 40 years. Missions to Kuiper Belt objects ( $>40 \mathrm{AU}$ ), the local interstellar medium beyond the heliopause (>150 AU), the Oort Cloud $(10000-50000 \mathrm{AU})$, and ultimately the nearest star system ( $\alpha$ Centauri at $270000 \mathrm{AU})$ were considered.

Present rocket technology falls short of the necessary propulsion requirements by orders of magnitude. Radically new capabilities are needed, and one approach is by extreme extrapolation of known technologies. Ideas presented at the workhop thus included: anti-matter initiated fusion; particle beams which could be accurately directed to a target vehicle at light-year distances owing to nanotechology navigation capabilities built into the particles themselves; current-carrying tether arrays 1000 by $1000 \mathrm{~km}$ in size that could generate Lorentz forces by interaction with the interstellar magnetic field; laser-pushed lightsails that could be accurately pointed and maintain collimation at stellar distances, etc.

The extreme sizes of structures and the extreme tolerances required for such concepts is worrisome. Taking the laser-driven sail as an example, let us assume that a mission propelled by a $1 \mathrm{~km}$ diameter lightsail 
is halfway $\left(2 \times 10^{13} \mathrm{~km}\right)$ to $\alpha$ Centauri when a beam problem reaches the vehicle. A one part in $10^{13}$ misalignment of the laser which occurred 2 years previously back on earth is now reaching the vehicle causing the beam to miss the light sail. Owing to the speed-of-light limitation, it will be another 2 years before any news of this transmitted by the spacecraft can reach the earth. It will be yet another 2 years before the correction from earth will reach the spacecraft. But by then the vehicle may have drifted out of its trajectory sufficiently owing to the secular effects of interaction with the interstellar medium (or other causes) that it is still out of the beam. Indeed, any drift of the vehicle from a line-of-sight trajectory will cause the same uncorrectible problem in the first place since there is no way to know where the vehicle is "now" (in the sense of where the beam is supposed to hit). This illustrates the inherent problem of speed-of-light caused time delay in any feedback loop; it would be all too easy - in fact probably unavoidable - to have a mission "lost in space without a paddle" due to the slightest error using this propulsion mechanism.

An alternative approach to extreme and perhaps unrealistic technologies is to consider what kinds of new physics might leapfrog us beyond this: after all, no amount of money and energy expended on maximizing information transmission via 19th century pony express couriers could approach the amount and instantaneity of information transmission by televison or the internet for example. In this case, the capabilities of "new physics" in electrodynamics utterly superseded mechanics.

Obviously one would like new physics that will permit faster-than-light $(v>c)$ travel and provide access to unlimited energy. The $v>c$ hope cannot be encouraged, however, owing to the fundamental conflicts it causes with respect to relativity and causality. ${ }^{a}$ However it does appear that there may be the equivalent of other new physics lurking within the electrodynamics of the quantum vacuum.

At this time there are four possibilities relevant to future propulsion technology that have a sufficiently welldeveloped basis in quantum vacuum physics so as to warrant further theoretical investigation: extraction of energy, generation of force, and manipulation of inertia and possibly even of gravitation. The realization of any one of these would leapfrog beyond all other concepts of interstellar travel presented at the workshop, and this was the topic of an invited presentation at the Caltech conference by Haisch.

\section{THE ZERO-POINT FIELD OF THE QUANTUM VACUUM}

A NASA-funded research effort has been underway since 1996 at the Lockheed Martin Advanced Technology Center in Palo Alto and at the California State University in Long Beach to explore the physics of the quantum vacuum and its possible long-term potential applications. That effort is a follow-on to previous work suggesting a relationship between the electromagnetic zero-point field of the quantum vacuum and inertia (Haisch, Rueda and Puthoff, 1994).

In the conventional interpretation of quantum theory, an electromagnetic zero-point field arises as a consequence of the Heisenberg uncertainty relation as applied to each mode of the electromagnetic field. No oscillator can ever be brought completely to rest due to quantum fluctuations. The minimum energy for a mechanical oscillator whose natural frequency is $\nu$ is $E=h \nu / 2$. Each mode of the electromagnetic field also acts as an oscillator. Thus for any frequency, $\nu$, direction, $\vec{k}$, and polarization state, $\sigma$, there is a minimum energy of $E=h \nu / 2$ in the electromagnetic field. Summing up all of these modes, each with its $E=h \nu / 2$ of energy, results in an electromagnetic ground state of energy that should permeate the entire universe: the

${ }^{a}$ In a famous speech in 1900, Lord Kelvin extolled the near completeness of physics (owing in no small measure, naturally, to his own Herculean efforts) with only two dark clouds on the otherwise clear horizon: the blackbody problem and the failure to detect the ether. At the moment there is arguably a glimmering of two even smaller clouds on the horizon with respect to relativity and causality that may ultimately point the way to some conceivable $v>c$ physics. With respect to relativity, it would be surprising if the rest frame defined by the cosmic microwave background did not turn out to be somehow special after all, perhaps opening the door to non-Lorentzian space-time physics. With respect to causality, the cloud is even wispier. It is an enigma within an anomaly that there is some credible evidence for human extrasensory perception and that this perception of information appears not to be dependent upon time, there apparently being no greater barrier to accessing future information than present information (Jahn et al. 1997). It would, of course, be unwise to make any interstellar plans on this basis. 
electromagnetic zero-point field, or ZPF. (The term ZPF refers to either the zero-point fields or equivalently zero-point fluctuations of the electromagnetic quantum vacuum; the term ZPE refers to the energy content of the electromagnetic quantum vacuum.) All other natural or artificial electromagnetic radiation would sit on top of this very energetic ground state.

The volumetric density of modes between frequencies $\nu$ and $\nu+d \nu$ is given by the density of states function $N_{\nu} d \nu=\left(8 \pi \nu^{2} / c^{3}\right) d \nu$. Using this density of states function and the minimum energy, $h \nu / 2$, that we call the zero-point energy per state one can calculate the ZPF spectral energy density:

$$
\rho(\nu) d \nu=\frac{8 \pi \nu^{2}}{c^{3}} \frac{h \nu}{2} d \nu
$$

It is instructive to write the expression for zero-point spectral energy density side by side with blackbody radiation:

$$
\rho(\nu, T) d \nu=\frac{8 \pi \nu^{2}}{c^{3}}\left(\frac{h \nu}{e^{h \nu / k T}-1}+\frac{h \nu}{2}\right) d \nu
$$

The first term (outside the parentheses) represents the mode density, and the terms inside the parentheses are the average energy per mode of thermal radiation at temperature $T$ plus the zero-point energy, $h \nu / 2$, which has no temperature dependence. Take away all thermal energy by formally letting $T$ go to zero, and one is still left with the zero-point term: the ground state of the electromagnetic quantum vacuum. The laws of quantum mechanics as applied to electromagnetic radiation force the existence of a background sea of zero-point-field $(\mathrm{ZPF})$ radiation.

Zero-point radiation is taken to result from quantum laws. It is traditionally assumed in quantum theory, though, that the ZPF can for practical purposes be ignored or subtracted away. The foundation of the discipline in physics known as stochastic electrodynamics (SED) is the exact opposite (see e.g. de la Peña and Cetto 1996 for a thorough review of SED). It is assumed in SED that the ZPF is as real as any other electromagnetic field. As to its origin, the assumption is that zero-point radiation simply came with the Universe. The justification for this is that if one assumes that all of space is filled with ZPF radiation, a number of quantum phenomena may be explained purely on the basis of classical physics including the presence of background electromagnetic fluctuations provided by the ZPF. The Heisenberg uncertainty relation, in this view, becomes then not a result of the existence of quantum laws, but of the fact that there is a universal perturbing ZPF acting on everything. The original motivation for developing SED was to see whether the need for quantum laws separate from classical physics could thus be obviated entirely.

Philosophically, a universe filled - for reasons unknown - with a ZPF but with only one set of physical laws (classical physics consisting of mechanics and electrodynamics), would appear to be on an equal footing with a universe governed - for reasons unknown - by two distinct physical laws (classical and quantum). In terms of physics, though, SED and quantum electrodynamics, QED, are not on an equal footing, since SED has been successful in providing a satisfactory alternative to only some quantum phenomena (although this success does include a classical ZPF-based derivation of the all-important blackbody spectrum, cf. Boyer 1984). Some of this is simply due to lack of effort: The ratio of man-years devoted to development of QED is several orders of magnitude greater than the expenditure so far on SED.

There is disagreement about whether this zero-point field should be regarded as real or virtual. A number of well-established phenomena such as the Casimir force and the Lamb shift are equally well explained in terms of either the action of a real ZPF or simply the quantum fluctuations of particles. This paradox is discussed in some detail by Milonni (1988). It is clearly essential to determine how "real" the zero-point field is.

\section{ENERGY EXTRACTION FROM THE QUANTUM VACUUM}

In the early part of this century the discovery of radioactivity seemed to violate the law of energy conservation. Heat and radiation appeared to be continuously given off as if by a source of "free energy" in certain elements (e.g. radium, uranium). The resolution came with the understanding that mass was being 
converted into energy via the $E=m c^{2}$ relationship of special relativity in a process of spontaneous decay of unstable elements, the naturally occuring radioisotopes. The low-level energy emitted by decay of natural or artificially-created radioisotopes is of limited use. However with the successful demonstration of fission in the 1940's, nuclear engineering became possible allowing us to tap a much more powerful mode of atomic energy release .

Chemical energy production, as in the burning of petroleum-based fuels or conventional rocket propulsion, taps the energy in the orbital electrons of atoms. Atomic energy generation taps the binding energy of the nuclear constituents of atoms. Is it possible to tap yet a deeper (and potentially much more powerful) source of energy: the ZPE of the electromagnetic quantum vacuum? There are two issues that bear on this.

It is often assumed that attempting to tap the energy of the vacuum must violate thermodynamics. One cannot extract thermal energy from a reservoir at temperature, $T_{1}$, if the environment is at temperature, $T_{2}>T_{1}$. While it is true that the ZPE is the energy remaining when all energy sources have been removed and the temperature reduced to $T=0 \mathrm{~K}$, the $\mathrm{ZPE}$ is not a thermal reservoir. It has very different characteristics than ordinary heat. The thermodynamics of energy extraction from the quantum vacuum have been analyzed by Cole and Puthoff (1993). They conclude as follows:

Relatively recent proposals have been made in the literature for extracting energy and heat from electromagnetic zero-point radiation via the use of the Casimir force. The basic thermodynamics involved in these proposals is analyzed and clarified here, with the conclusion that, yes, in principle, these proposals are correct. Technological considerations for the actual application and use are not examined here, however.

If the zero-point field is a real electromagnetic ground state, then there is no inconsistency with present-day physics in the possibility of tapping this energy. Indeed, it has been proposed that certain astrophysical processes are driven by the natural extraction of such energy (cf. Rueda, Haisch and Cole 1995 and references therein) and an ideal experiment has been proposed by Forward (1984) that clearly demonstrates the conceptual possibility of extracting vacuum energy.

A second point to consider is that the orbital energy of electrons and the $E=m c^{2}$ relationship itself may both ultimately be traceable to zero-point energy. While limited so far to the single, simple case of the ground-state of hydrogen, the work of Boyer (1975) and Puthoff (1987) suggests that electron energy levels may be stabilized against radiative collapse by interaction with the quantum vacuum. This would in principle link chemical energy to the energy of the ZPF.

In his preliminary development of the Sakharov conjecture on gravity as a ZPF-induced force, Puthoff (1989) suggests that the $E=m c^{2}$ relationship reflects the kinetic energy of zitterbewegung (Schrödinger's term) which originates in the fluctuations induced by the ZPF on charged particles (quarks and electrons). In other words, instead of expressing a relationship between mass and energy, the $E=m c^{2}$ relationship tells us how much ZPF-driven energy is associated with a given particle. When this energy is liberated it is thus not really a transformation of mass into energy, rather a release of zero-point energy associated with this quantum motion known as zitterbewegung. This would in principle link atomic energy to the energy of the ZPF. (An attempt at a classical visualization of this motion from the SED viewpoint may be found in Rueda, 1993).

If the above interpretations prove to be valid and it is ultimately the energy of the ZPF that is being tapped in chemical and atomic energy production processes, then it is not inconceivable that other channels will be found to liberate energy from the quantum vacuum. Since the ZPF must be universal, a propulsion drive energized by the ZPF would have access to unlimited "fuel" anywhere... the ZPE thus providing the ultimate energy source.

\section{IN-SITU GENERATION OF FORCES}

The existence of the Casimir force - an attraction between uncharged conducting plates - is now well established. Measurements by Lamoreaux (1997) are in agreement with theoretical predictions to within a few percent. A particularly simple interpretation involving the ZPF was presented by Milonni, Cook and Goggin (1988): 
We calculate the vacuum-field radiation pressure on two parallel, perfectly conducting plates. The modes outside the plates push the plates together, those confined between the plates push them apart, and the net effect is the well-known Casimir force.

In other words, the electromagnetic boundary conditions on the two plates exclude a certain amount of ZPF from the cavity in between. This results in an overpressure from the ZPF outside, which then acts as a pressure on the plates.

This is one interpretation: call it the ZPF radiation pressure model. It is also possible to interpret the force as "a macroscopic manifestation of the retarded van der Waals force between two neutral polarizable particles" (Milonni, Cook and Goggin 1988), i.e. a quantum effect involving the particles in the plates (see Milonni 1982).

The speculation concerning propulsion is that the ZPF radiation pressure model of the Casimir force is physically correct and that it may be possible to construct some wall or cavity that interacts with the ZPF differently on one side than on the other. If that were possible, one would have in effect a ZPF-sail that could provide a propulsive force anywhere in space.

\section{MODIFICATION OF INERTIAL AND GRAVITATIONAL MASS}

The ultimate capability enabling practical interstellar travel would be the ability to tap the energy of the vacuum while at the same time modifying the inertia of the spacecraft. Reducing inertia of a spacecraft would allow higher velocity for the same expenditure of energy and more rapid acceleration without damage to the structure owing to the reduction of inertial forces. The absolute limit would be acceleration to velocity $c-\epsilon$ in time $\delta$ where both $\epsilon$ and $\delta$ approach zero.

Until recently there was absolutely no basis in physics for even considering such a possibility. While such a possibility still appears to be remote, there now does exist a basis in physics to at least begin to explore this concept.

In 1994 Haisch, Rueda and Puthoff published a paper, "Inertia as a zero-point field Lorentz Force," in which a substantive mathematical analysis indicated that the inertia of matter could be interpreted as an electromagnetic reaction force originating in the quantum vacuum. This concept has now been redeveloped by Rueda and Haisch $(1998 \mathrm{a}, 1998 \mathrm{~b})$ in a way that is both mathematically simpler while at the same time yielding a properly covariant relativistic result. This is encouraging that we are on the right track.

In a frame at rest or in uniform motion, the ZPF is uniform and isotropic. This is due to the Lorentz invariance of the ZPF spectrum. (The spectral cutoff of the spectrum would not be Lorentz invariant, but if the inertia interaction takes place at a frequency or resonance far from the cutoff, this would not matter.) However in an accelerated frame the ZPF becomes asymmetric. Rueda and Haisch have shown that the Poynting vector - which characterizes the radiative energy flux - becomes non-zero in an accelerating frame. If the quarks and electrons in matter undergoing acceleration scatter this ZPF flux, a reaction force will arise that is proportional to accleration. This is proposed to be the origin of inertia of matter. Inertia is not an innate property of matter; it is an acceleration-dependent electromagnetic reaction force.

The principle of equivalence mandates that gravitational and inertial mass must be the same. Therefore, if inertial mass is electromagnetic in origin, then gravitational mass must also be electromagnetic in some fashion. A preliminary development of a gravitational analysis based on the electrodynamics of the ZPF has been made by Puthoff (1989). Subsequent critiques have pointed out some deficiencies in this analysis. Nevertheless, it is encouraging that the ZPF-related parameters that determine "mass" turn out to be identical in the inertia and gravitation analyses (see Appendix A in Haisch, Rueda and Puthoff 1994). From this view, the $\mathrm{ZPF}$ acts as a mediator of a gravitational force, but cannot itself gravitate, hence would not result in an unacceptably large cosmological constant (Haisch and Rueda 1997).

We now have a theoretical basis to explore the possibility that electrodynamics may be used to modify the quantum vacuum in some way so as to alter inertia and/or gravitation. It would be prudent to continue such investigations. 


\section{ACKNOWLEDGMENTS}

We acknowledge support of NASA contract NASW-5050 for this work. BH also acknowledges the hospitality of Prof. J. Trümper and the Max-Planck-Institut where some of these ideas originated during several extended stays as a Visiting Fellow. AR acknowledges stimulating discussions with Dr. D. C. Cole.

\section{REFERENCES}

Boyer, T. H., "Random electrodynamics: The theory of classical electrodynamics with classical electromagnetic zero-point radiation," Phys. Rev. D, 11, 790 (1975).

Boyer, T. H., "Derivation of the blackbody radiation spectrum from the equivalence principle in classical physics with classical electromagnetic zero-point radiation," Phys. Rev. D, 29, 1096 (1984).

Cole, D.C. and Puthoff, H.E., "Extracting energy and heat from the vacuum," Phys. Rev. E, 48, 1562 (1993).

de la Peña, L. and Cetto, A.M. The Quantum Dice: An Introduction to Stochastic Electrodynamics, Kluwer Acad. Publishers, Dordrecht, (1996).

Forward, R., "Extracting electrical energy from the vacuum by cohesion of charged foliated conductors," Phys. Rev. B, 30, 1700 (1984).

Haisch, B. and Rueda, A., "Reply to Michel's 'Comment on Zero-Point Fluctuations and the Cosmological Constant'," Astrophys. J., 488, 563 (1997).

Haisch, B., Rueda, A. and Puthoff, H.E. (HRP), "Inertia as a zero-point-field Lorentz force," Phys. Rev. A, 49, 678 (1994).

Jahn, R.G, Dunne, B.J., Nelson, R.D., Dobyns, Y.H. and Bradish, G.J., "Correlations of Random Binary Sequences With Pre-stated Operator Intention: A Review of a 12-year Program," J. Scientific Exploration, 11, 345 (1997).

Lamoreaux, S.K., "Demonstration of the Casimir Force in the 0.6 to $6 \mu \mathrm{m}$ Range," Phys. Rev. Letters, 78, 5 (1997)

Milonni, P.W., "Casimir forces without the vacuum radiation field," Phys. Rev. A, 25, 1315 (1982).

Milonni, P.W., "Different Ways of Looking at the Electromagnetic Vacuum," Physica Scripta, T21, 102 (1988).

Milonni, P.W., Cook, R.J. and Goggin, M.E., "Radiation pressure from the vacuum: Physical interpretation of the Casimir force," Phys. Rev. A, 38, 1621 (1988).

Puthoff, H.E., "Ground state of hydrogen as a zero-point-fluctuation-determined state," Phys. Rev. D, 35, 3266 (1987).

Puthoff, H.E., "Gravity as a zero-point-fluctuation force," Phys. Rev. A, 39, 2333 (1989).

Rueda, A., "Stochastic Electrodynamics with Particle Structure: Part I - Zero-point induced Brownian Behaviour," Found. Phys. Letters, 6, 75 (1993); and "Stochastic Electrodynamics with Particle Structure: Parts II - Towards a Zero-point induced Wave Behaviour," 6, 193 (1993).

Rueda, A. and Haisch, B., "Inertia as reaction of the vacuum to accelerated motion," Physics Letters A, 240, 115 (1998a).

Rueda, A. and Haisch, B., "Contribution to inertial mass by reaction of the vacuum to accelerated motion," Foundations of Physics, 28, 1057 (1998b).

Rueda, A., Haisch, B. and Cole, D. C., "Vacuum Zero-Point Field Pressure Instability in Astrophysical Plasmas and the Formation of Cosmic Voids," Astrophys. J., 445, 7 (1995). 